Received: 2012.12 .20

Published: 2013.05.22
Corresponding Author:

\title{
Influence of CPAP treatment on inflammation in patients with obstructive sleep apnea: Is it a matter of time or a matter of marker?
}

\author{
Zhen-hua Gao \\ Yong-zhong Yang
}

Zhen-hua Gao, e-mail: tjgzh125@yahoo.cn
Department of Cardiology, Han Dan Central Hospital, Han Dan People's Republic of China

It was with a great interest that we have read the article entitled "Effect of CPAP treatment on endothelial function and plasma CRP levels in patients with sleep apnea" by Athanasios Panoutsopoulos et al. published recently in this journal [1]. After reading the article, we benefited a lot from it. In recent years our department has been studying the relationship between inflammation, coronary heart disease and sleep apnea. Athanasios Panoutsopoulos and coworkers encouraged us greatly. We would like to share our opinions about the study. The study demonstrated that Continuous positive airway pressure (CPAP) treatment had a relevant upon systemic and local inflammation and provided new insight into pathophysiological pathways of obstructive sleep apnea syndrome (OSAS).

OSAS was widely accepted as a cardiovascular risk factor. The close association of OSAS and conventional cardiovascular risk factors including hypertension, diabetes mellitus, dyslipidemia and obesity added to the adverse cardiovascular complication.

Studies have suggested a strong bidirectional association between OSAS and metabolic syndrome (MS). Inflammation was believed to play a role in the pathogenesis of cardiovascular disease.

OSAS was closely associated with inflammation, but obesity may be a confounding factor. Obesity has emerged as one of the most prevalent diseases in the world. The mechanisms leading to obesity-related morbidities were likely multifactorial, and absolutely included activation of inflammatory pathways. Both obesity and OSAS could modulate the expression and secretion of systemic and airway inflammatory cytokines. So, we should pay certain attention to the change of weight.

CPAP was effective in the treatment of OSAS, but adherence was a major barrier. The subjects completed the CPAP treatment with good adherence in this study. Several studies reported that CPAP treatment could reverse pathophysiological changes, reduce the risk of cardiovascular events and mortality in subjects with OSAS. C-reactive protein (CRP) was a prototypic marker of inflammation that has repeatedly shown promise as a potentially reliable biomarker of cardiovascular morbidity.

Hypertension induced by OSAS may be multifactorial in origin, and systemic inflammation was one of the major factors. OSAS patients with hypertension have higher level of inflammation and insulin resistance.

Sleep apnea was common in patients with coronary artery disease (CAD). Inflammatory processes played an important role in the pathogenesis of atherosclerosis. Inflammation may be linked to CAD progression by accelerating atherosclerotic processes. Statins has been widely utilized to reduce cardiovascular risk in patients with cardiovascular disease. The benefit has been attributed both to cholesterol lowering and "anti-inflammatory" effects. Smoking was a known risk factor and was reported to be associated with an increased prevalence of OSAS. Due to the smoking-induced upper airway inflammation and stimulant effects of nicotine on upper airway, smoking may participate in the pathophysiology of OSA.

Some studies confirmed that physical exercise could improve excessive daytime sleepiness due to its improvement in inflammatory profiles.

\section{Conflict of interest}

The author has no personal or financial support or author involvement with organization(s) with financial interest in the subject matter or any conflict of interest to report.

\section{References:}

1. Panoutsopoulos A, Kallianos A, Kostopoulos K et al: Effect of CPAP treatment on endothelial function and plasma CRP levels in patients with sleep apnea. Med Sci Monit, 2012; 18(12): 747-51

Full-text PDF: http://www.medscimonit.com/download/index/idArt/883918

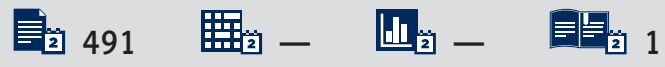

